\title{
Electron scattering and acceleration by a tightly focused laser beam
}

\author{
Yousef I. Salamin, ${ }^{*}$ Guido R. Mocken, and Christoph H. Keitel \\ Theoretische Quantendynamik, Fakultät für Physik, Universität Freiburg, Hermann-Herder-Strasse 3, D-79104 Freiburg, Germany
} (Received 31 January 2002; published 18 October 2002)

\begin{abstract}
By numerically solving the relativistic equations of motion of a single electron in laser fields modeled by those of a Gaussian beam, we demonstrate electron capture by, reflection from, and transmission through the beam. In modeling the fields, terms of order up to $\epsilon^{5}$, where $\epsilon$ is the diffraction angle, are retained. All cases of capture are accompanied by energy gain that may reach a few $\mathrm{GeV}$, from fields of present-day intensities. Reflection and transmission, on the other hand, result sometimes in no gain or even in a loss of energy. It is shown that a laboratory static magnetic field may be used to eject a captured electron, a process that sometimes results in even more energy gain. For example, a $2.5 \mathrm{~T}$ uniform magnetic field suffices to eject a $3.633 \mathrm{MeV}$ electron injected at $6^{\circ}$ to the axis of a linearly polarized beam of a $10 \mathrm{PW}$ power output and aimed at a point near the focus. Such an electron gains $1128 \mathrm{MeV}$ from the laser field alone. However, it emerges with a $1230 \mathrm{MeV}$ net energy gain under the additional action of the small magnetic field.
\end{abstract}

DOI: 10.1103/PhysRevSTAB.5.101301

PACS numbers: 42.65.-k, 42.50.Vk, 52.75.Di

\section{INTRODUCTION}

Recent advances in the technology of intense laser fields [1-4] continue to motivate research efforts into the issue of electron acceleration [5-14], aiming at the realization of a high energy gain over a short distance of interaction $[15,16]$. Wang and co-workers $[7,10]$ have recently demonstrated the phenomenon of electron capture, accompanied by energy gain, in numerical simulations modeling the laser fields by those of a lowest-order Gaussian beam. They have also investigated variation of the maximum energy gain with the various beam parameters and initial conditions on the electron injection. Issues of similar nature have also been the subject of a recent Letter [17].

A few questions arise in this context. We would like to know in what ways the electron energy gain may be altered if terms of higher order in the diffraction angle are included when the fields of the beam are modeled, and whether an electron that has been captured can be easily extracted from the beam. It is also of interest to know whether such an electron would lose part or all of its energy gain from interaction with such fields, once it is extracted. Furthermore, it would be quite interesting to know if aiming the electron right at the focus, where the fields are believed to be the strongest, will always result in the highest possible energy gain.

These issues have been addressed by two of us in a recent Letter [18] in which the tightly focused laser beam has been accurately modeled. In that model, however, the maximal laser intensity was kept the same for all laser spot sizes $w_{0}$ employed. Obviously, high $w_{0}$ values require high laser powers prior to focusing and, hence, some of the relevant results may be considered of little practical utility. We remove this shortcoming by expressing the intensity parameter in terms of the laser power output in the important calculations of the present paper.
In addition, we give a detailed study of the model used including an outline of the derivation and a discussion of the fields. Extraction of the electron from the interaction region near the beam focus, using a suitably applied uniform magnetic field, has been shown in this Letter to be accompanied by the loss of part of the energy gained. Here, we give an explicit example in which the converse is true, as well, depending upon the size of the region over which the dc magnetic field is applied.

This paper presents results of numerical investigations of the dynamics, mainly trajectory and energy gain, of a single electron injected at a finite angle to the direction of propagation of a Gaussian laser beam and aimed at points on the beam axis within a few Rayleigh lengths on either side of the stationary beam focus. The paper tackles several issues of interest in this field, including the demonstration of reflection, transmission, and, more importantly, capture and violent acceleration to $\mathrm{GeV}$ energy, all when corrections to the fields include terms of the order of 5 in the diffraction angle. Many examples are presented in this paper which constitute ample evidence that electron acceleration, from a few $\mathrm{MeV}$ to several $\mathrm{GeV}$, is possible using present-day laser field intensities. Energy gain is found to result, with varying magnitudes, in situations corresponding to reflection, transmission, and capture. While capture always results in gain, reflection and transmission accompanied by gain are less common. It will be demonstrated that a small static magnetic field, applied in the direction of the main laser magnetic field component, is sufficient to give the electron a little nudge and eject it out of the more intense regions of the beam. The gain drops or increases somewhat, as expected, at the end of this process due to Compton collisions suffered by the electron.

In Sec. II the electron dynamics in a plane wave is briefly reviewed. This is followed in Sec. III by a short 
discussion of the fields of a Gaussian beam including higher-order corrections. Details of the analytic calculation leading to the fields will be relegated to the Appendix. We start Sec. IV by explaining our approach in the investigations to follow. The issue of injection into points on the beam axis on both sides of the focus will be taken up next, and inclusion of higher-order terms in modeling the fields is finally shown to estimate the energy gain more reliably. In Sec. V we show, via an example, how an appropriately applied uniform magnetic field may be used to extract a captured electron while increasing its energy gain by about $9 \%$. In Sec. VI the energy gain dependence upon the laser frequency variations is discussed. Finally our conclusions will be given in Sec. VII.

\section{THE PLANE-WAVE TREATMENT}

Our terminology will be introduced in this section, together with the electron-field configuration and a brief review of the electron interaction with the fields of a plane wave [19-22]. Let $m$ and $-e$ be the mass and charge of the electron, respectively. We will consider electron injection at an angle $\theta_{i}$ relative to the propagation direction $\mathbf{k}$ of the linearly polarized laser beam. The direction of polarization will be taken along $x$ in the system of coordinates shown in Fig. 1. Without any loss of generality, the initial direction of the electron motion will be taken in the $x z$ plane, henceforth to be referred to as the $(\mathbf{k}, \boldsymbol{E})$ plane. In other words, the initial velocity vector will be

$$
\beta_{0}=\beta_{0}\left(\hat{\mathbf{i}} \sin \theta_{i}+\hat{\mathbf{k}} \cos \theta_{i}\right)
$$

where $\beta_{0}$ is the initial speed normalized by $c$, the speed of light in vacuum. We use $\hat{\mathbf{i}}, \hat{\mathbf{j}}$, and $\hat{\mathbf{k}}$ to denote unit vectors in the positive $x, y$, and $z$ directions, respectively. In this (a)

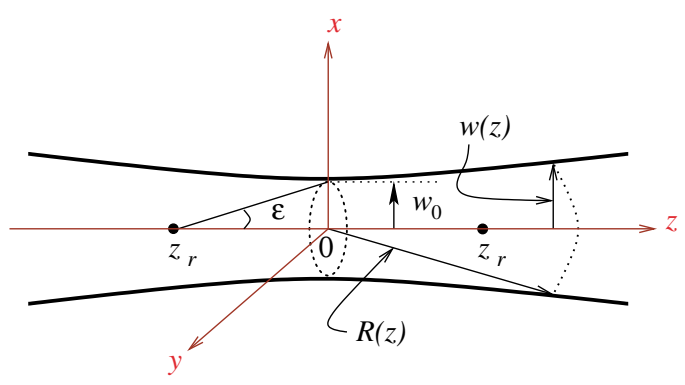

(b)

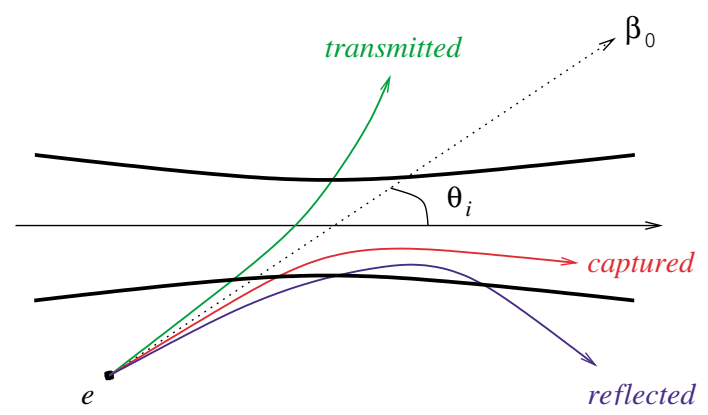

FIG. 1. (Color) (a) Geometry of the Gaussian beam. (b) A schematic diagram of the electron motion.

section, the electron will be initially aimed at the origin of coordinates from the initial position denoted by $\mathbf{r}_{0}$ relative to the same coordinate origin.

In general, the electron relativistic dynamics will be discussed in terms of an energy-momentum 4-vector $(\mathcal{E} / c, p)$, where the energy is $\mathcal{E}=\gamma m c^{2}$ and the momentum is $\mathbf{p}=\gamma m c \beta$, with the Lorentz factor given by $\gamma=$ $\left(1-\beta^{2}\right)^{-1 / 2}$, and $\beta$ is the velocity scaled by $c$. Describing the laser beam fields in terms of a transverse vector potential $\mathbf{A}(\eta)$, the electron trajectory will be given, as a function of the plane-wave phase $\eta=\omega t-$ $k z$, by the position vector $[21,22]$

$$
\mathbf{r}(\eta)=\mathbf{r}_{0}+\frac{\lambda}{2 \pi}\left\{\int_{\eta_{0}}^{\eta}\left[\frac{\gamma_{0} m c \beta_{0}+\frac{e}{c} \mathbf{A}\left(\eta^{\prime}\right)}{\gamma_{0} m c\left(1-\hat{\mathbf{k}} \cdot \boldsymbol{\beta}_{0}\right)}\right] d \eta^{\prime}+\hat{\mathbf{k}} \int_{\eta_{0}}^{\eta}\left[\frac{\frac{1}{2}\left(\frac{e \mathbf{A}\left(\eta^{\prime}\right)}{\gamma_{0} m c^{2}}\right)^{2}+\left(\frac{e \mathbf{A}\left(\eta^{\prime}\right)}{\gamma_{0} m c^{2}}\right) \cdot \beta_{0}}{\left(1-\hat{\mathbf{k}} \cdot \boldsymbol{\beta}_{0}\right)^{2}}\right] d \eta^{\prime}\right\},
$$

where $\lambda$ is the radiation field wavelength. Furthermore, the particle's energy may be given by

$$
E(\eta)=\gamma_{0} m c^{2}\left[1+\frac{\frac{1}{2}\left(\frac{e \mathbf{A}(\eta)}{\gamma_{0} m c^{2}}\right)^{2}+\left(\frac{e \mathbf{A}(\eta)}{\gamma_{0} m c^{2}}\right) \cdot \beta_{0}}{\left(1-\hat{\mathbf{k}} \cdot \beta_{0}\right)}\right] .
$$

In Eqs. (2) and (3), and throughout this work, a zero subscript will mean an initial value at $t=0$ of the subscripted quantity.

Consider a plane-wave representation of the field in terms of the linearly polarized vector potential

$$
\mathbf{A}(\eta)=\hat{\mathbf{i}} A_{0} \cos \eta
$$

Throughout this work, the laser field peak intensity, $I_{0}$, will be given in terms of the dimensionless parameter $q=e A_{0} /\left(m c^{2}\right)$, where $I_{0} \lambda^{2}=1.375 \times 10^{18} q^{2}$, when $I_{0}$ is expressed in $\mathrm{W} \mathrm{cm}^{-2}$ and $\lambda$ in $\mu \mathrm{m}$. An example electron trajectory, calculated using Eq. (2), is shown in Fig. 2(a). The electron-field interaction commences at $t=0$ at the point $\mathbf{r}_{0}=\left(z_{0} \tan \theta_{i}, 0, z_{0}\right)$ and motion is followed for five field cycles. Here, one field cycle is defined as a change of $2 \pi$ in $\eta$. In Fig. 2(b) the energy gain, defined as

$$
\text { energy gain }=\mathcal{E}(\eta)-\gamma_{0} m c^{2}=\left(\gamma-\gamma_{0}\right) m c^{2}
$$

is shown for the same example. Note, for now, that according to this simple picture the electron moves continuously inside the laser beam; i.e., it is captured. Extraction of the electron out of the beam is not theoretically well defined. In addition to that the electron is shown to exchange a tremendous amount of energy with the field. Any energy gain, however, is temporary [21-23]. 

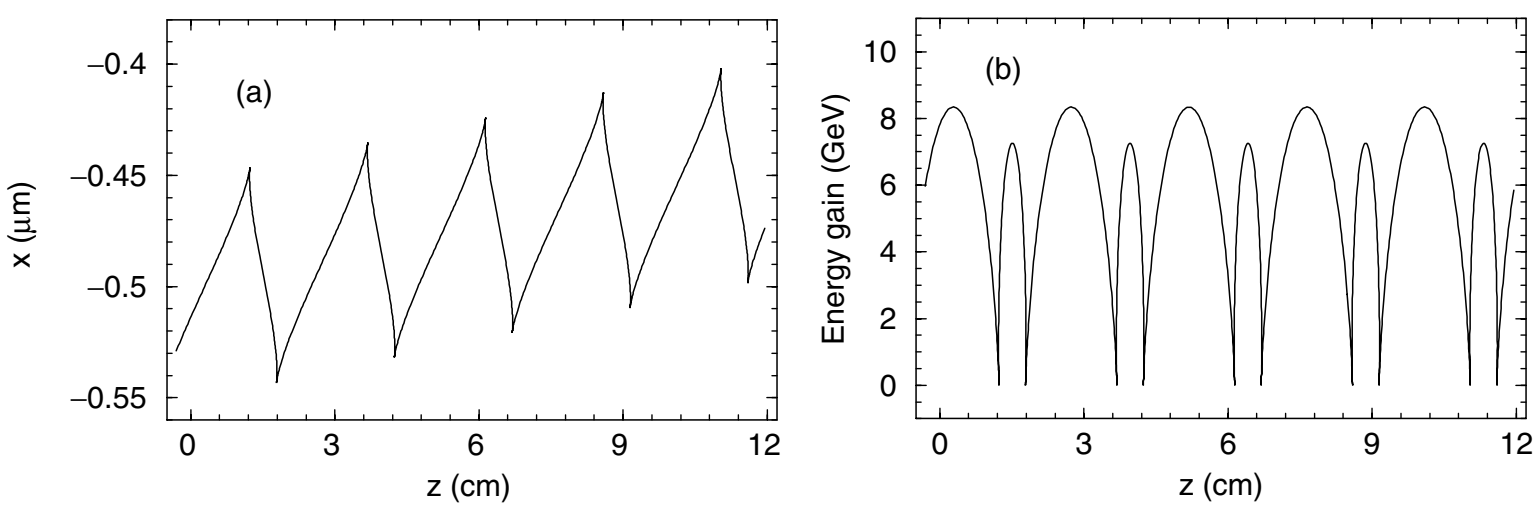

FIG. 2. (a) Electron trajectory in a plane wave field, calculated on the basis of Eq. (2). (b) Electron energy gain during interaction with the same field, calculated using Eqs. (3) and (5). The beam parameters are $\lambda=1.056 \mu \mathrm{m}$ and $q=100$. The electron parameters are $\gamma_{0}=20, \theta_{i}=10^{\circ}$, and $z_{0}=-3 \mathrm{~mm}$. Duration of interaction corresponds to five field cycles, or $\Delta \eta=10 \pi$.

We conclude this section by observing that, while it is analytically tractable, and hence, it allows one to build a clear intuitive picture, the plane-wave treatment is only approximate. Among its virtues also is the fact that it helps one to design suitable numerical codes that need ultimately be used in precise calculations that model the fields more realistically.

\section{THE FIELDS NEAR A GAUSSIAN BEAM FOCUS}

Since we intend to investigate the electron dynamics in the relativistic regime of laser intensities, and because the required high intensity-laser fields can only be realized by focusing over small dimensions, a detailed knowledge of the laser electric and magnetic fields near the focus of the Gaussian beam is essential [24-28].

In what follows, the beam axis is taken along $z$, with its propagation direction along $+z$ and stationary focus at $z=0$. The beam cross section at focus is circular and has a radius $w_{0}$; a cross section at an arbitrary $z$ is also circular with radius $w(z)=w_{0} \sqrt{1+\left(z / z_{r}\right)^{2}}$. Furthermore, $z_{r}=k w_{0}^{2} / 2$ is the Rayleigh length, and $\epsilon=$ $w_{0} / z_{r}$ is the diffraction angle. Now, letting $\xi=x / w_{0}$, $v=y / w_{0}$, and $\zeta=z / z_{r}$, the electric components of the laser field associated with such a beam, to order $\epsilon^{5}$, are $[24,25,27]$ (see also the Appendix)

$$
\begin{gathered}
E_{x}=E\left\{S_{0}+\epsilon^{2}\left[\xi^{2} S_{2}-\frac{\rho^{4} S_{3}}{4}\right]+\epsilon^{4}\left[\frac{S_{2}}{8}-\frac{\rho^{2} S_{3}}{4}-\frac{\rho^{2}\left(\rho^{2}-16 \xi^{2}\right) S_{4}}{16}-\frac{\rho^{4}\left(\rho^{2}+2 \xi^{2}\right) S_{5}}{8}+\frac{\rho^{8} S_{6}}{32}\right]\right\}, \\
E_{y}=E \xi v\left\{\epsilon^{2}\left[S_{2}\right]+\epsilon^{4}\left[\rho^{2} S_{4}-\frac{\rho^{4} S_{5}}{4}\right]\right\}, \\
E_{z}=E \xi\left\{\epsilon\left[C_{1}\right]+\epsilon^{3}\left[-\frac{C_{2}}{2}+\rho^{2} C_{3}-\frac{\rho^{4} C_{4}}{4}\right]+\epsilon^{5}\left[-\frac{3 C_{3}}{8}-\frac{3 \rho^{2} C_{4}}{8}+\frac{17 \rho^{4} C_{5}}{16}-\frac{3 \rho^{6} C_{6}}{8}+\frac{\rho^{8} C_{7}}{32}\right]\right\} .
\end{gathered}
$$

Similarly, the magnetic field components are given by

$$
\begin{gathered}
B_{x}=0, \\
B_{y}=E\left\{S_{0}+\epsilon^{2}\left[\frac{\rho^{2} S_{2}}{2}-\frac{\rho^{4} S_{3}}{4}\right]+\epsilon^{4}\left[-\frac{S_{2}}{8}+\frac{\rho^{2} S_{3}}{4}+\frac{5 \rho^{4} S_{4}}{16}-\frac{\rho^{6} S_{5}}{4}+\frac{\rho^{8} S_{6}}{32}\right]\right\}, \\
B_{z}=E v\left\{\epsilon\left[C_{1}\right]+\epsilon^{3}\left[\frac{C_{2}}{2}+\frac{\rho^{2} C_{3}}{2}-\frac{\rho^{4} C_{4}}{4}\right]+\epsilon^{5}\left[\frac{3 C_{3}}{8}+\frac{3 \rho^{2} C_{4}}{8}+\frac{3 \rho^{4} C_{5}}{16}-\frac{\rho^{6} C_{6}}{4}+\frac{\rho^{8} C_{7}}{32}\right]\right\} .
\end{gathered}
$$

In Eqs. (6)-(11), we have taken

$$
\begin{gathered}
E=E_{0} \frac{w_{0}}{w} g(\eta) \exp \left[-\frac{r^{2}}{w^{2}}\right], \\
S_{n}=\left(\frac{w_{0}}{w}\right)^{n} \sin \left(\psi+n \psi_{G}\right),
\end{gathered}
$$

$$
C_{n}=\left(\frac{w_{0}}{w}\right)^{n} \cos \left(\psi+n \psi_{G}\right)
$$

Furthermore, $k=\omega / c, k A_{0}=E_{0}, r^{2}=x^{2}+y^{2}$, and $\rho=$ $r / w_{0}$. For a continuous beam with a stationary focus we 

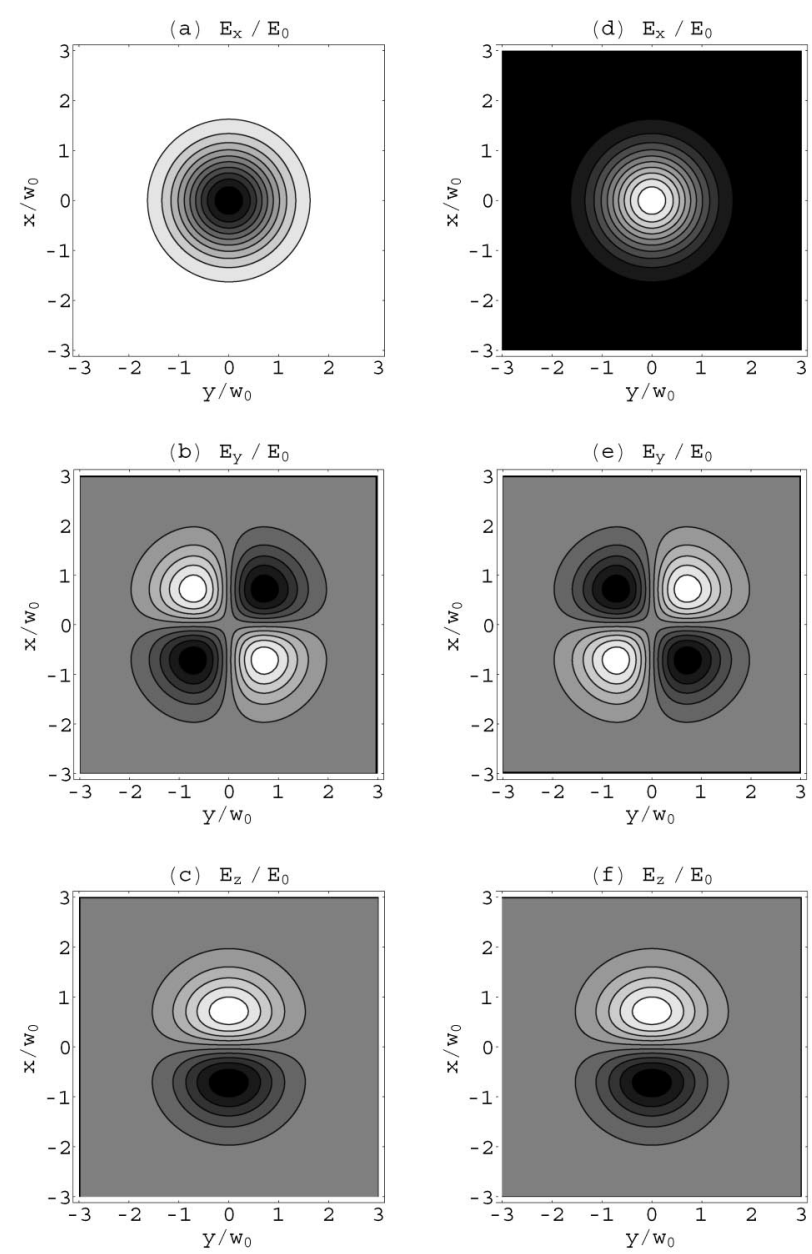

FIG. 3. Contour maps of the electric components of the laser field of a Gaussian beam over a square $30 \mu \mathrm{m}$ on a side, centered at the beam focus and extending in the $x y$ plane. The beam parameters are $w_{0}=5 \mu \mathrm{m}$ and $\lambda=1.056 \mu \mathrm{m}$. In (a) -(c) the fields are given at $\omega t=0$, and in (d)-(f) at $\omega t=$ $\pi / 2$. Note that positive and negative values are distinguished by the level of shading, dark means negative values and bright positive.

will take $g(\eta)=1$ in this work. Some of the details of the calculation leading to Eqs. (6)-(11) are collected in the Appendix. See also Fig. 1(a). These equations were derived from a vector potential polarized along $x$, with an amplitude $A_{0}$, and a frequency $\omega$. The remaining symbols in Eqs. (6)-(11) have the following definitions:

$$
\begin{gathered}
\psi=\psi_{0}+\psi_{P}-\psi_{R}+\psi_{G}, \\
\psi_{P}=\omega t-k z, \\
\psi_{G}=\tan ^{-1} \zeta, \\
\psi_{R}=\frac{k r^{2}}{2 R},
\end{gathered}
$$

$$
R(z)=z+\frac{z_{r}^{2}}{z}
$$

Note that $\psi_{0}$ is a constant, $\psi_{P}=\eta$ is the plane-wave phase, $\psi_{G}$ is the Guoy phase associated with the fact that a Gaussian beam undergoes a total phase change of $\pi$ as $z$ changes from $-\infty$ to $+\infty, \psi_{R}$ is the phase associated with the curvature of the wave fronts, and that $R(z)$ is the radius of a curvature of a wave front intersecting the beam axis at the coordinate $z$. The fields given above satisfy Maxwell's equations $\boldsymbol{\nabla} \cdot \boldsymbol{E}=0=\boldsymbol{\nabla} \cdot \boldsymbol{B}$, plus terms of order $\epsilon^{6}$ [23].

Retention of terms of order $\epsilon^{2}$ and higher in the field equations brings about the small, but nonzero, component $E_{y}$, besides corrections of the same order of magnitude to the terms already present to order $\epsilon$. It will be shown that these terms and corrections play an important role in the electron capture and acceleration to high energy.

In Figs. 3 and 4 we show contour maps of the electric and magnetic fields in a plane perpendicular to the beam axis and crossing it at the focus. The maps are snapshots taken at $t=0$ and, later, at $t=\pi /(2 \omega)$. Bright spots correspond to positive values and dark areas represent negative ones. So an, otherwise free, electron will feel a force of attraction and, hence, be accelerated in dark regions and vice versa.

Needless to say that the fields are rapidly changing functions of the time and so are the field strength and sign at any one point. Moreover, where the electron will be at any point in time depends also upon its injection parameters, namely, the initial position $\left(x_{0}, 0, z_{0}\right)$, the initial injection energy $\gamma_{0}$, and the initial injection
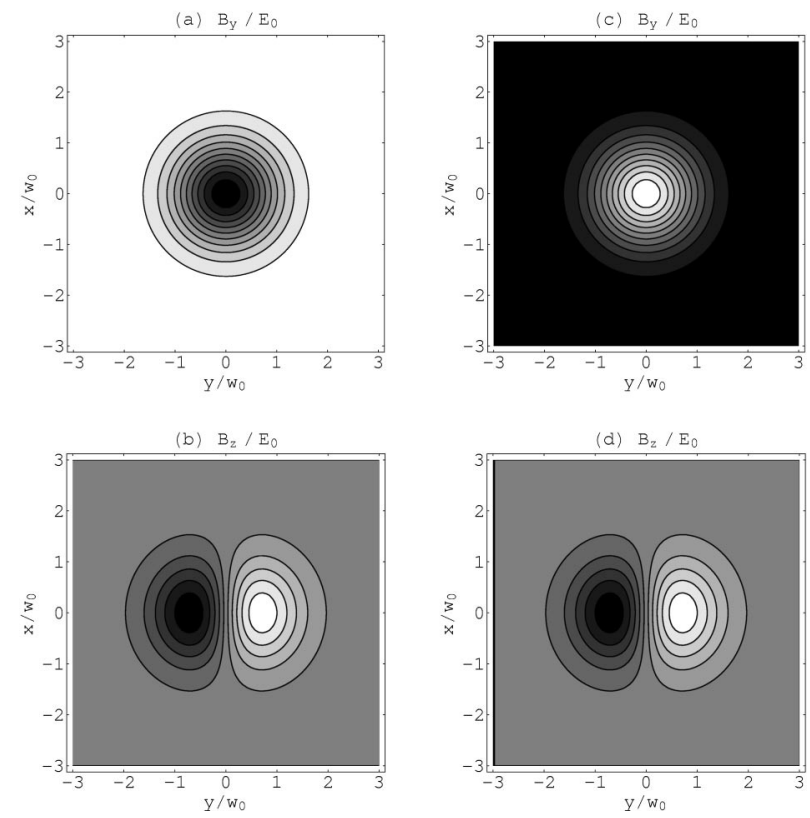

FIG. 4. Same as Fig. 3, but for the magnetic components. (a) and (b) give the fields at $\omega t=0$, and (c) and (d) at $\omega t=\pi / 2$. 

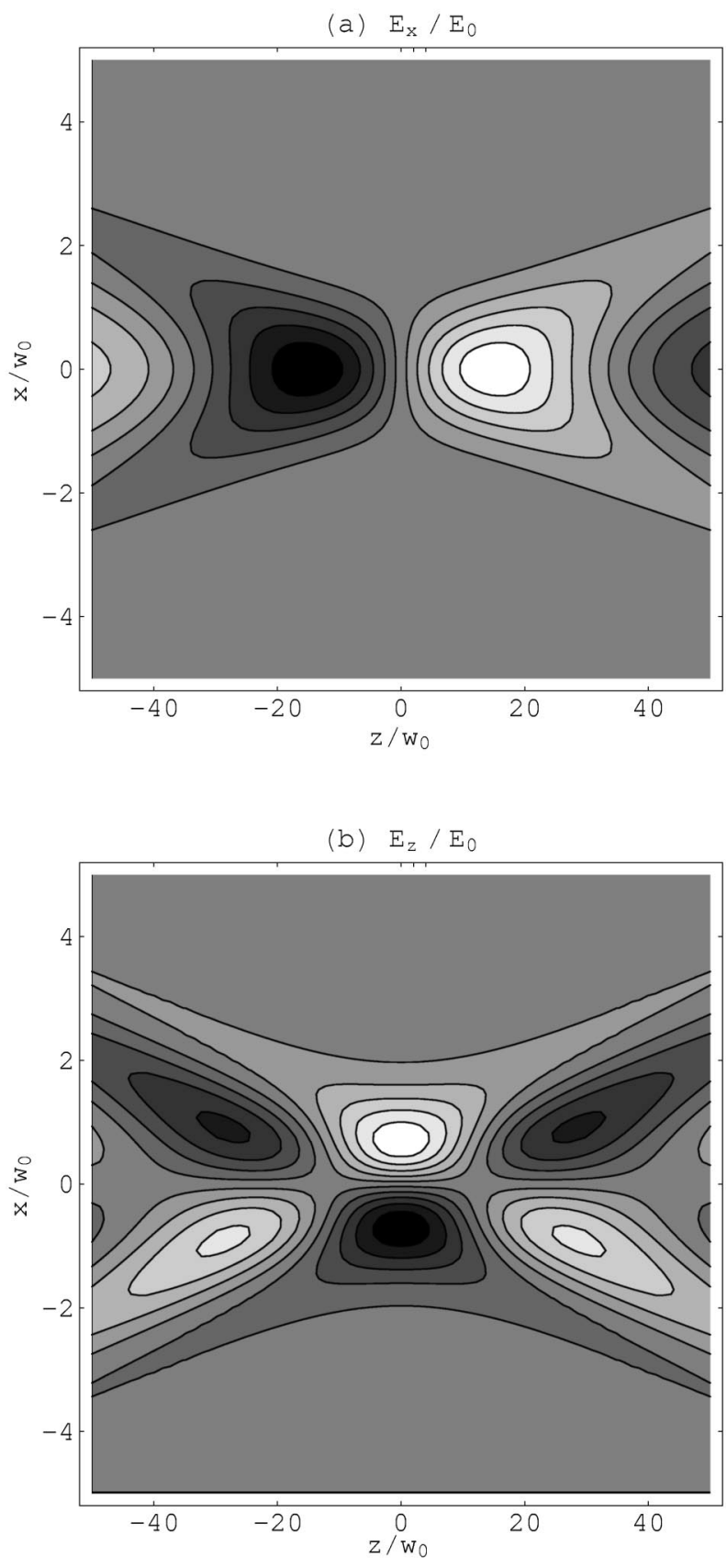

FIG. 5. Contour plots of the $E_{x}$ and $E_{z}$ components over part of the polarization plane. These are snapshots corresponding to $\omega t=0$ and for field parameters the same as in Fig. 3.

angle $\theta_{i}$. Other parameters that play a role in determining the ensuing dynamics include $w_{0}, q$, and $\psi_{0}$.

Roughly speaking, since $E_{x}$ and $B_{y}$ are the largest field components, the ensuing electron motion will most strongly be influenced by them. For the high field intensities of interest to us in this work, $q \gg 1$, motion out of the $(\mathbf{k}, \boldsymbol{E})$ plane turns out to be small. So, only projection of the electron trajectory onto this plane will be considered. On the other hand, $E_{x}$ and $E_{z}$ will play the biggest part in producing the energy gain we are after. Figure 5 shows a contour plot of these field components in a portion of the $(\mathbf{k}, \boldsymbol{E})$ plane around the focus. Note that, if aimed sideways at an angle to the beam axis and especially towards a point on either side of the focus, the electron samples parts of the beam lacking symmetry. This increases its chances of gaining energy.

\section{ELECTRON DYNAMICS IN A GAUSSIAN LASER BEAM}

\section{A. General considerations}

Dynamics of the electron, in the fields given above, is governed by the equations

$$
\begin{gathered}
\frac{d \mathbf{p}}{d t}=-e(\mathbf{E}+\beta \times \mathbf{B}), \\
\frac{d \mathcal{E}}{d t}=-e c \beta \cdot \mathbf{E} .
\end{gathered}
$$

Any energy gain or loss takes place at a time rate governed by Eq. (21), which now consists of three contributions, one from each component of $\boldsymbol{E}$. On the other hand, $\boldsymbol{\beta}$ results from integration of the force Eq. (20) which, in turn, involves contributions from all three electric field force components, as well as both components of the $\boldsymbol{\beta} \times \boldsymbol{B}$ or ponderomotive force. Relative magnitude of the contribution to the energy gain, or acceleration, from each of these five force components may be estimated with the help of Eqs. (6)-(11).

Complexity of the equations of motion leaves little room for further analytic manipulation and help in drawing an intuitive picture. We will therefore resort to solving the equations numerically throughout this work.

We illustrate the general procedure and give preliminary comments on some of the results, via the examples shown in Fig. 6. In this figure trajectories, momentum transfers, and energy gains are shown for three sets of parameters. In Fig. 6(a) parts of the actual trajectories in the full fields are given, along with the spatial extension of the beam boundaries as background. Strictly speaking, it is not accurate to talk about a boundary for a laser beam, but for definiteness, we will adopt the curves in the (k, $\boldsymbol{E}$ ) plane of the functions $x= \pm w(z)$ as giving the loci of points of intersection of a beam surface with that plane. On those lines the field intensity falls to $e^{-2}$ of its maximum value on the beam axis. Typically, an electron trajectory starts from the injection point with coordinates $\left(z_{0}, x_{0}\right)=\left(-5,-5 \tan \theta_{i}\right) \mathrm{mm}$, heads towards the focus, interacts with the beam and gets reflected, captured, or transmitted. Note that, in the immediate neighborhood of the focus, the electron trajectory has features similar to those of the plane-wave case displayed in Fig. 2(a). This should come as no surprise, as the wave fronts in that region of a Gaussian beam are almost planar. According to Eq. (19) the radius of curvature $R \rightarrow \infty$ as $z \rightarrow 0$. 

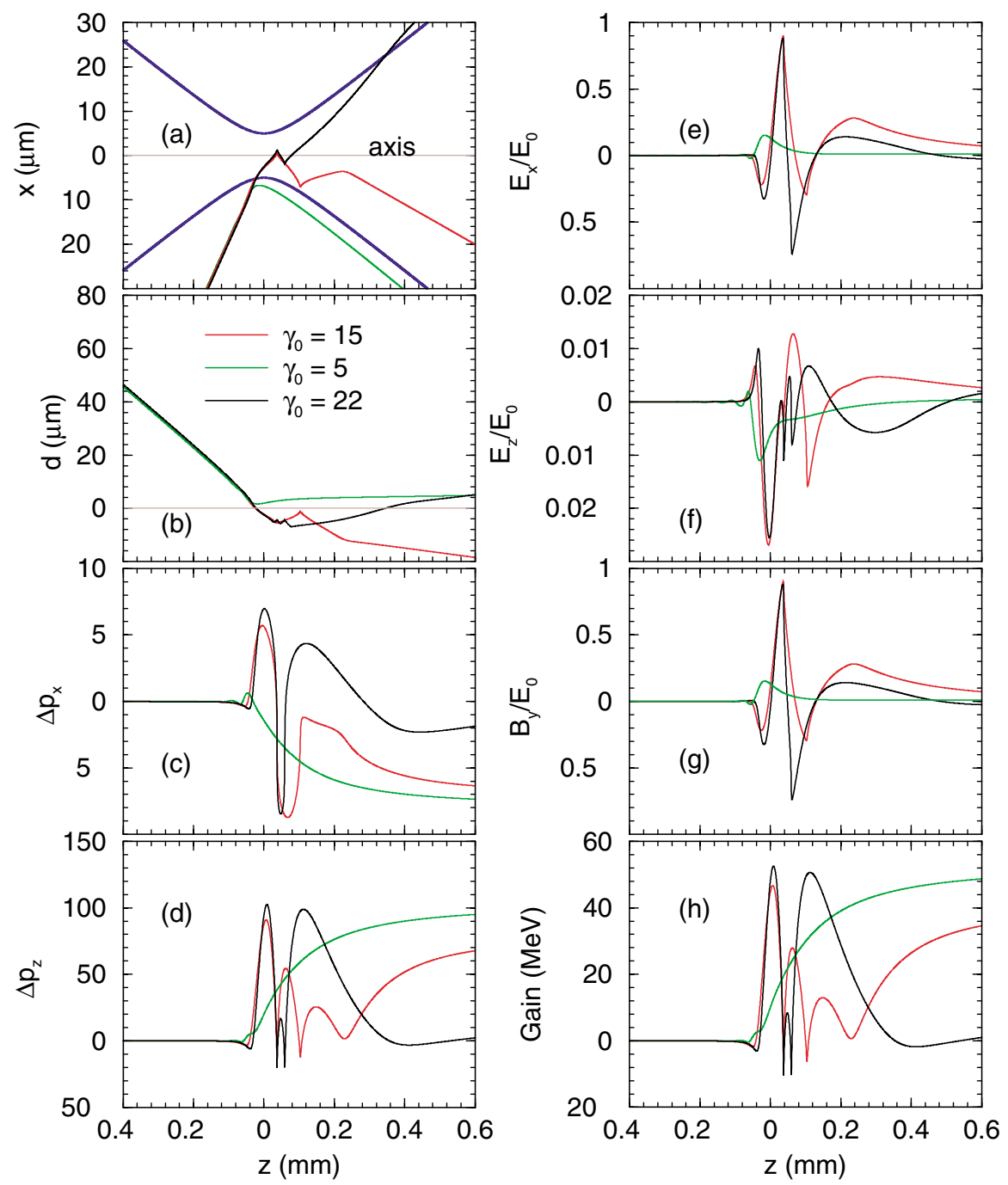

FIG. 6. (Color) Examples illustrating the cases of reflection (green), capture (red), and transmission (black). Only a small region around the beam focus is shown in each case. (a) The trajectories. (b) The quantity $d$ defined by Eq. (22). (c) The electron $x$ momentum transfer $\Delta p_{x}=\gamma \beta_{x}-\gamma_{0} \beta_{0} \sin \theta_{i}$. (d) The electron $z$ momentum transfer $\Delta p_{z}=\gamma \beta_{z}-\gamma_{0} \beta_{0} \cos \theta_{i}$. (e) - (g) The field components $E_{x}, E_{z}$, and $B_{y}$, respectively, sensed by the electron along its trajectory. (h) The electron energy gain. For all cases considered, $z_{0}=-5 \mathrm{~mm}, \theta_{i}=10^{\circ}, q=$ $10, w_{0}=5 \mu \mathrm{m}, \lambda=1 \mu \mathrm{m}$, and the initial injection energy is $\gamma_{0}$. At the end of the total interaction time equivalent to $10^{5}$ laser field cycles, or $\omega t=3.10135 \times 10^{6}$ the gains are 43.2 MeV (reflection), $34.5 \mathrm{MeV}$ (capture), and $4 \mathrm{MeV}$ (transmission). Note that the legends in (b) apply everywhere else in this figure.

To distinguish between capture, on the one hand, and reflection and transmission, on the other, we define a capture parameter, $d$, by

$$
d=|x|-w(z) .
$$

Obviously, the electron will be captured by the beam during that part of the motion for which $d<0$; otherwise it will be either reflected or transmitted. What we have decided to label as the beam boundary are points for which $d=0$ or points at which the field amplitude in Eq. (12) falls to $1 / e$ of its value on the beam axis. Figure 6(a) demonstrates all three cases visually, and 6(b) lends more support to the distinctions made between them on the basis of the simple test just described. More on this issue may be found in Sec. IV D below.

According to the quantum mechanical particle picture, three Compton processes compete, depending upon the field intensity range, in accounting for the electron-field interaction. Those are normal, nonlinear, and stimulated Compton scattering. In a classical treatment one may distinguish only between elastic and inelastic processes, by investigating the momentum transfers. In Figs. 6(c) and 6(d) such transfers are shown 
for the electrons whose trajectories are given in 6(a). The figures show a lot of momentum exchange between the electron and the field while the former is in the immediate vicinity, and a little to the right, of the focus. A little more momentum transfer takes place far away from the focus. Thus the subsequent electron acceleration is a result of momentum transferred to the electron from the radiation field over a short time, i.e., in the nature of a strong impulse.

Figures 6(e)-6(g) show plots of the field components $E_{x}, E_{z}$, and $B_{y}$ sensed by the electron along its trajectory for the examples considered above. Note first that $E_{z}$ is typically some 2 orders of magnitude smaller than $E_{x}$ and that $E_{x} \sim B_{y}$, in the Gaussian system of units we are using. It is not hard to notice the lack of symmetry in the field oscillations (not to be confused with the field cycles). Thus interaction of the electron with a negative portion of, say $E_{x}$, results in energy gain. Subsequent interaction with the following positive portion does not result in total loss of the gained energy, and so on. This may be seen by studying 6(e) and 6(h) together. Furthermore, by studying a typical trajectory portion given in Fig. 6(a) together with the corresponding fields in $6(\mathrm{e})-6(\mathrm{~g})$ one can easily understand the correlation between, say, the turning points on a typical trajectory, and the points at which, say $E_{x}$, ceases to increase (or decrease), and so on.

Finally, in Fig. 6(h) the electron energy gain, defined by Eq. (5) and calculated numerically here, is shown. We stress that the examples shown in Fig. 6 have been carefully chosen for illustrative purposes and thus the energy gains in 6(h) are very modest. More impressive results will be shown shortly.

\section{B. Injection on both sides of the focus}

In all cases considered so far, the electron was aimed directly at the beam focus $(0,0,0)$. This has been done because the intention was to let the electron sample the regions of highest field intensity. It turns out that this may not lead to the best energy gain possible. Because of other parameters in the configuration, the focus, too, may present the electron with strong, but symmetric, accelerating fields over small dimensions. Whereas on both sides, the fields occupy a much larger volume and the electronfield interactions take place over longer times. Besides, in that region a full field cycle consists of two asymmetric half cycles. Thus energy gained during interaction with one-half of a field cycle does not get completely lost during interaction with the following half cycle.

Figure 7 shows the variation of the energy gain with the coordinate $z$ value, denoted by $s$, of the point on the axis towards which the electron is initially aimed. In all cases the injection angle is fixed at $\theta_{i}=5^{\circ}$, and the $z$ coordinate of the injection point is fixed at $z_{0}=-3 \mathrm{~mm}$, while
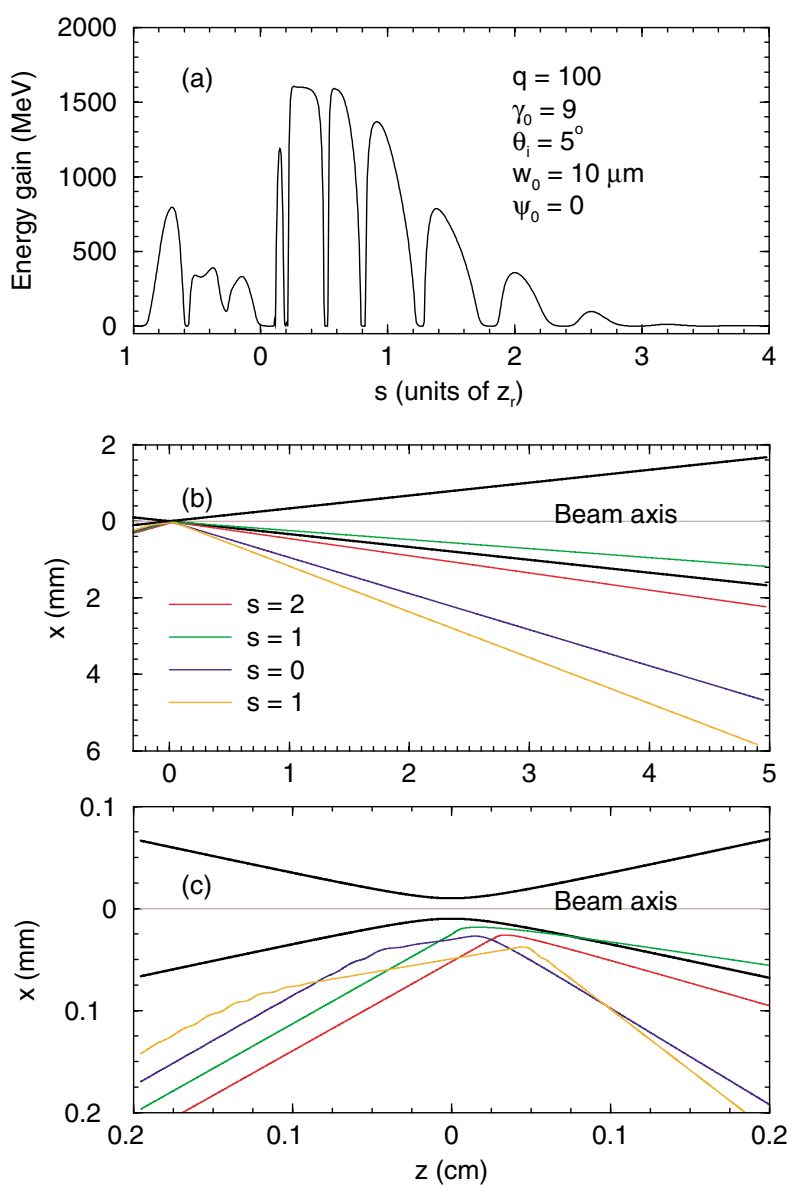

FIG. 7. (Color) For an electron with initial velocity vector pointing from $\left(x_{0}, 0, z_{0}=-3 \mathrm{~mm}\right)$ to $(0,0, s)$ the following are shown: (a) variation of the energy gain with $s$, (b) the actual trajectories of four cases corresponding to different values of $s$, and (c) a magnified portion of (b) around the laser beam focus. The laser wavelength is $\lambda=1.056 \mu \mathrm{m}$. Note that, in this configuration, $x_{0}=-\left(s-z_{0}\right) \tan \theta_{i}$. See Fig. 1(a). The interaction time, in all cases, is such that $\omega t=\pi \times 10^{5}$ and terms of the order of 5 in the diffraction angle have been used to model the fields. In (b) and (c) the dark lines mark the beam boundaries. The legends in (b) apply to (c) as well.

the $x$ coordinate of that point is calculated from $x_{0}=$ $-\left(s-z_{0}\right) \tan \theta_{i}$. The highest gain found, for the chosen parameter set, is about $1.6 \mathrm{GeV}$. Note that the gain goes down as one moves the target point farther out and away from the focus, where the intensity drops markedly in the downstream regions of the beam. Out of the trajectories, shown in Figs. 8(a) and 8(b), and selected from among the cases displayed in 7(a), only one corresponds to a weakly captured electron, while the others are reflected. As already noted above, this high gain is attributed to the fact that, along each trajectory, the electron samples asymmetric regions of the beam, in addition to encountering stronger fields there compared to points far away from the focus. Conversely, when aimed at points like the ones immediately to the right of the focus in this 

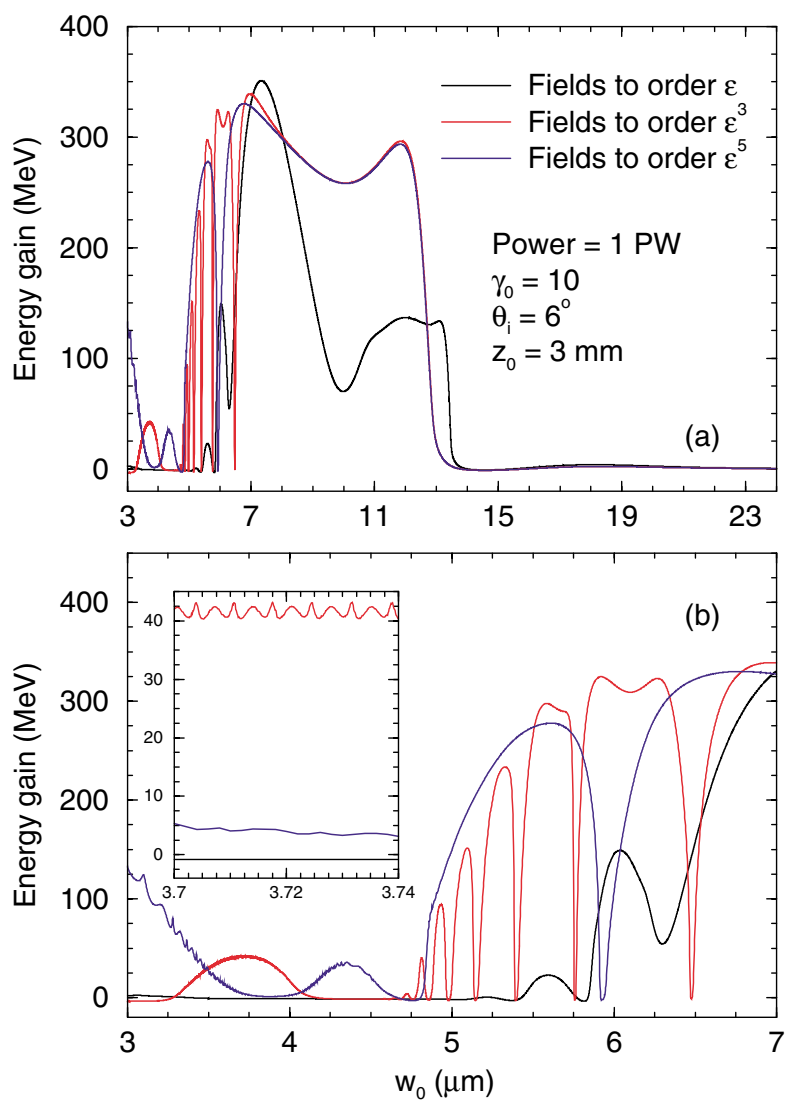

FIG. 8. (Color) Energy gain vs the beam waist radius $w_{0}$ with the laser output power fixed. Injection is into the beam focus and $\psi_{0}=0$. Note that in (b) we zoom in on a small part of (a) and that the inset in (b) shows yet a smaller portion of the same data. The legends and parameter values in (a) apply to the other figures as well.

example, the electron acquires little or no gain at all. We have examined the fields sensed by the electron (not shown here) for a number of such points and have found that they exhibit high symmetry, in the sense described above.

A laser system is often characterized by its output power $P$. For the fields given by Eqs. (6)-(11) the power may be calculated by integrating the time-averaged Poynting vector over a plane through the beam focus and perpendicular to its axis. Dropping terms in the result of order $\epsilon^{6}$ and smaller, one gets

$$
\begin{aligned}
P[T W] & =\frac{\pi w_{0}^{2}}{2} I_{0}\left[1+\frac{\epsilon^{2}}{4}+\frac{\epsilon^{4}}{8}\right], \\
& \approx 0.0216\left(\frac{q w_{0}}{\lambda}\right)^{2}\left[1+\frac{\epsilon^{2}}{4}+\frac{\epsilon^{4}}{8}\right],
\end{aligned}
$$

where $I_{0}=I(0,0,0)=c E_{0}^{2} / 8 \pi$ is the peak intensity (at the focus). Equation (23) clearly shows that for a fixed laser output power, the peak intensity is inversely proportional to the square of the beam waist radius, or equiv- alently $q$ is inversely proportional to $w_{0}$. The need to focus the beam over small spatial dimensions raises questions about the modeling of the tightly focused beam in ascending powers of the diffraction angle. In particular, it is desirable to know how adequate a certain representation is. In Fig. 8 we show the energy gain as a function of the beam radius at focus for three field representations containing terms that go as $\epsilon^{N}$, where $\epsilon$ is the diffraction angle and $N=1,3$, and 5 . For the set of parameters shown, one can easily distinguish between three regions. In region $R_{1}$ for which $w_{0}>15 \mu \mathrm{m}$ all three representations yield little net energy gain, as the fields sensed by the electron in this regime become highly symmetrical, just like those of a plane wave. In $R_{2}$, or for $7<w_{0}<$ $15 \mu \mathrm{m}$, the gain from the representation in terms of fields containing $\epsilon^{5}$ contributions differs only slightly from the gain calculated from a representation that stops at $\epsilon^{3}$. In the region $R_{3}$, where $w_{0}<7 \mu \mathrm{m}$, the $\epsilon^{3}$ model shows substantial deviations from that of the $\epsilon^{5}$ model in many places [see the inset of Fig. 8(b)].

To sum up, for the given parameter set, Fig. 8 has a number of messages to present. First, In region $R_{1}$, it suffices to represent the fields by retaining terms of order $\epsilon$. Second, terms of up to $\epsilon^{3}$ are sufficient in region $R_{2}$. Third, in $R_{3}$ one has to use terms of order $\epsilon^{5}$. Fourth, a representation in terms of fields that go only as $\epsilon$ is not reliable for a beam focused to a radius $w_{0}<15 \mu \mathrm{m}$. Finally, it appears that further corrections, beyond $\epsilon^{5}$, are needed when focusing is to go below $w_{0} \sim 4 \mu \mathrm{m}$.

\section{Beyond the paraxial approximation}

This is a good point to investigate the question of whether retaining terms in the fields of order $\epsilon^{3}$ and higher makes any difference at all when the electron is injected into points on both sides of the beam focus. To this end we have calculated the gain as a function of the distance $s$ defined above [see Fig. 7(a)], once using fields up to order $\epsilon$, i.e., those of a lowest-order Gaussian beam, once with fields of order $\epsilon^{3}$, and another time when the corrections of up to order $\epsilon^{5}$ are included. This is shown in Fig. 9, where the general features of the gain vs $s / z_{r}$ plots look the same as in Fig. 7(a). Obvious differences also exist (compare, for example, the regions around the focus in the two figures). As expected, based on the discussion of Fig. 8, since the value of $w_{0}=8 \mu \mathrm{m}$, i.e., the regime in question corresponds to region $R_{2}$, and the $\epsilon^{5}$ corrections lead to infinitesimal corrections to the gain.

The most important conclusion to be drawn from Fig. 9 is that the order $\epsilon$ terms alone do not adequately describe a Gaussian beam focused to $8 \mu \mathrm{m}$. To better appreciate this point, note that $\epsilon=\lambda / \pi w_{0}$. For Fig. 9, this gives $\epsilon \sim$ $1 / 8 \pi$. Viewed as an expansion parameter, this value of $\epsilon$ is not small enough. In other words, for such a small beam 


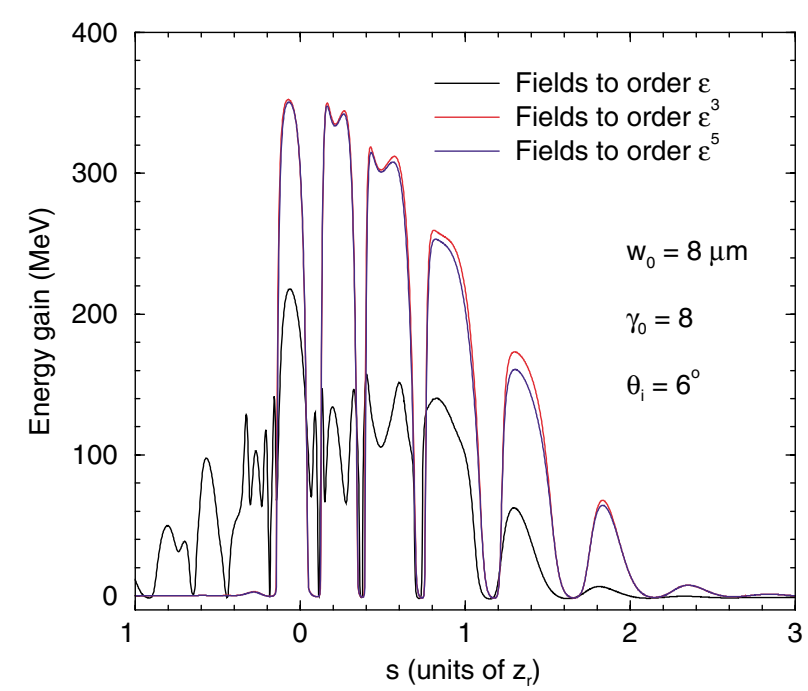

FIG. 9. (Color) Variation of the energy gain with $s$ when contributions to the fields of order up to $\epsilon^{N}, N=1,3$, and 5, are taken into account. Electron initial position is $\left[x_{0}=-(s-\right.$ $\left.\left.z_{0}\right) \tan \theta_{i}, 0, z_{0}=-3 \mathrm{~mm}\right]$ and it is injected into the point $(0,0, s)$. The laser wavelength is $\lambda=1 \mu \mathrm{m}$, and its output power is $P=1 \mathrm{PW}$.

radius at focus, one ought to include terms of order $\epsilon^{3}$. Note that inclusion of the $\epsilon^{5}$ field terms does not lead to appreciable corrections to the results based on the $\epsilon^{3}$ ones.

\section{Role of the injection energy}

In any scattering event each of several factors plays an important role in determining the outcome. In the remainder of this section, the sensitive role played by the injection energy is considered. On the one hand, an initially slow electron will not be able to penetrate the high intensity regions of the beam and may be reflected with little or no energy gain. On the other hand, an initially fast electron may pass through undeviated and gain or lose a small amount of energy. To be more specific we have plotted the gain vs the scaled injection energy in Fig. 10, for two different laser output powers. The situation corresponding to the peak of each hump shown is obtained when the electron senses a maximally asymmetric set of fields. This leads to maximum gain. The opposite happens at the minima on either side of a particular hump. Figure 10 has another purpose. Based on information derived from it one is able to determine approximately the range of injection energies that would result in a desired range of energy gains. This will be used, for example, in the next section.

It is desirable to try and derive a criterion or more, relating the initial injection energy to the laser field power or intensity, that would enable us to sharply distinguish between these events. One such criterion [20]
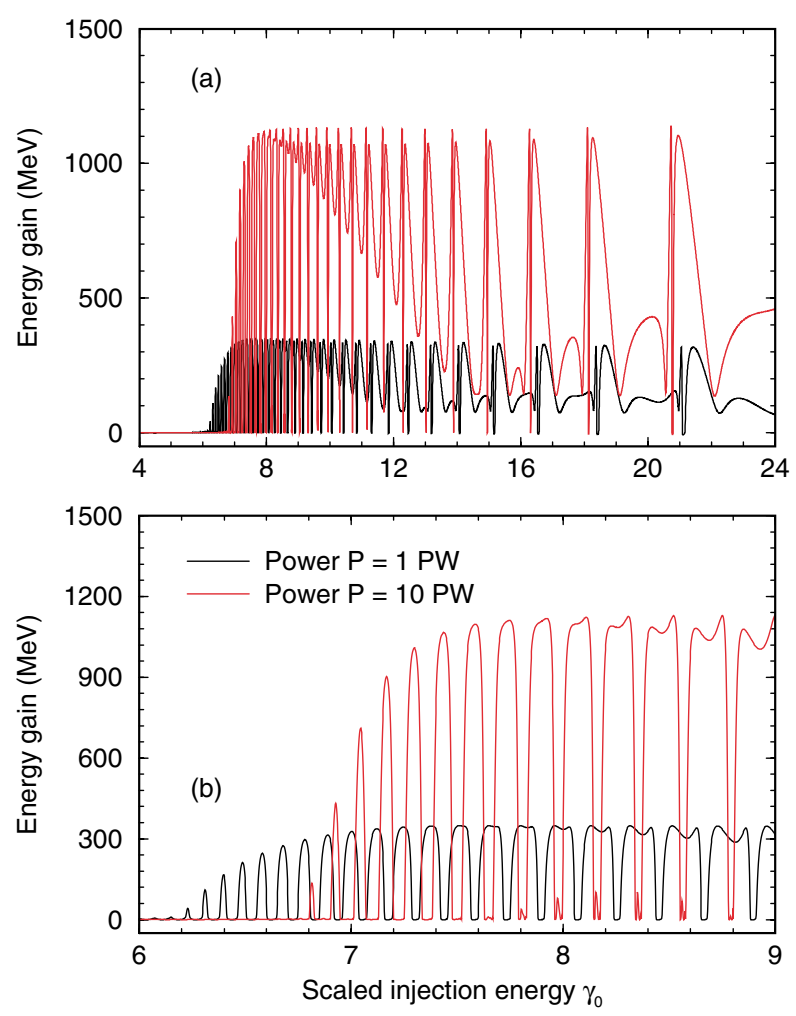

FIG. 10. (Color) Electron energy gain vs the scaled injection energy. In this case the electron is aimed at a point on the beam axis a distance $s=z_{r} / 5$ to the right of the focus. The laser wavelength is $\lambda=1 \mu \mathrm{m}$ and the intensity parameters are $q \sim$ 26.9 (output power $1 \mathrm{PW}$ ) and $q \sim 85$ (power $10 \mathrm{PW}$ ). The other parameters are $w_{0}=8 \mu \mathrm{m}, \theta_{i}=6^{\circ}, z_{0}=-3 \mathrm{~mm}$, and $\psi_{0}=$ 0 . Fields to order $\epsilon^{5}$ have been used in the calculation. Note that in (b) we merely zoom in on a small part of (a) and that the legends in (b) apply to (a) as well.

may be arrived at by replacing the spot size at focus, $w_{0}$, in the expression for the laser output power given by Eq. (23), with the maximum amplitude of transverse motion in the plane-wave result (2). A second criterion has recently been stated based on numerical investigations [10] according to which capture occurs if the field intensity exceeds the magnitude corresponding to $q=100$. Elsewhere Wang et al. [17] argue that capture should occur as long as the electron transverse initial momentum is much less than $q m c / \sqrt{2}$. Equivalently, Kibble [29] showed, a long time ago, that an electron injected sideways into the focus of a laser beam will be reflected as long as its injection kinetic energy $K=\left(\gamma_{0}-\right.$ 1) $m c^{2}$ is small compared to the ponderomotive energy $U_{p}=\left(e E_{0}\right)^{2} / 4 m \omega^{2}$. Unfortunately, all these criteria apply, if at all, to injection into the focus and would be valid only over the extremely small region over which the fields may be approximately plane wave in character. They are also rendered useless by the sensitive dependence of the motion and energy gain on the local phase variations, as will be demonstrated shortly. Ultimately each one of them 
results in a single approximate inequality whose truth value loses meaning due to the sensitive dependence upon the phase variations (or oscillations).

The oscillations exhibited in Fig. 10 are a manifestation of the rapid phase variations in the fields. One question that immediately comes to mind is whether the phase variations play any role in determining the outcome of any given event, i.e., whether it is going to be one of reflection, capture, or transmission. Suppose one determined that a certain injection energy $\gamma_{0}$ resulted in the electron being reflected. The question as to when the next capture event is going to happen, as one increases the injection energy beyond $\gamma_{0}$, cannot be answered easily. This is the case precisely because of the rapid local phase variations. To illustrate this point, we show in Fig. 11 the transverse electron coordinate $x^{*}$, at the end of the interaction time, as a function of the initial scaled injection energy for cases centered on the reflection event of Fig. 6. As a background, we also show $\pm w^{*}$ (see caption) calculated for every value of $\gamma_{0}$ used, evaluated also at the end
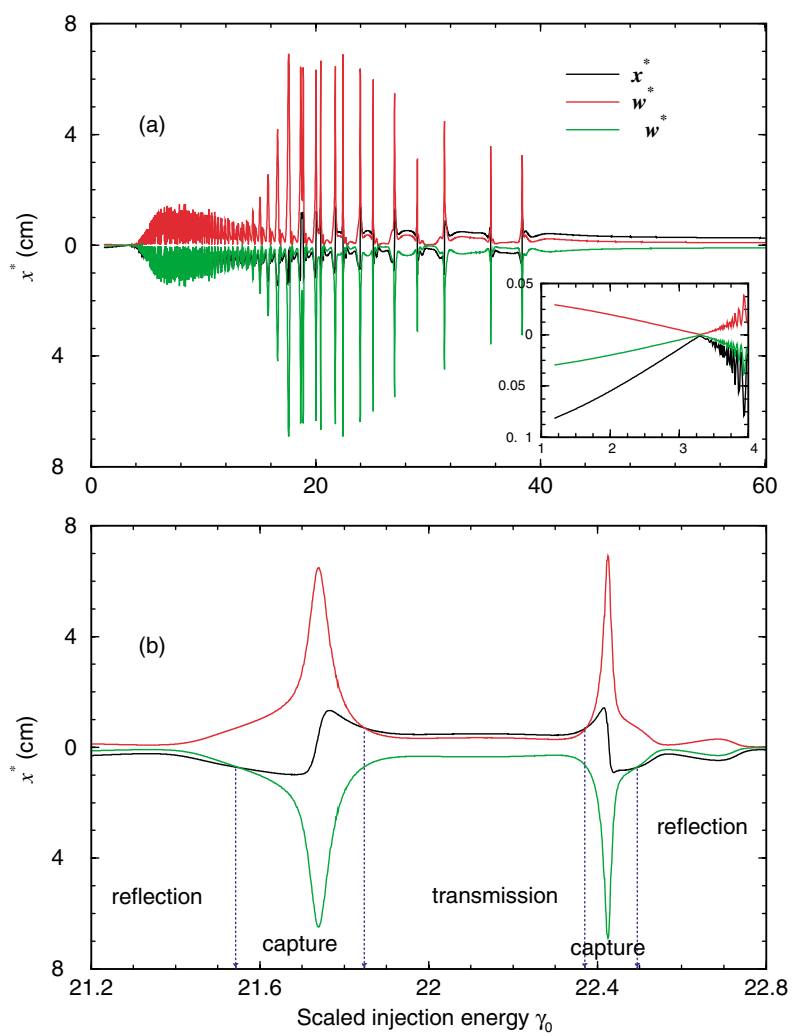

FIG. 11. (Color) Transverse electron coordinate $x^{*}$ (black curve), at the end of the interaction time, vs the scaled injection energy. Shown in red and green, as a background to help in classifying the events, are the corresponding values of $\pm w^{*}$, where $w^{*}$ is the radius of the beam cross section at the end of the interaction time corresponding to $\omega t=3.10135 \times 10^{6}$. The range of injection energies in (b) is centered on $\gamma_{0}=22$ shown in Fig. 6. All parameters are the same as in Fig. 6 and the legends in (a) apply to (b) as well. of the interaction time. We adopt the following classification scheme for the events

$$
\begin{cases}x^{*}<-w^{*}, & \text { (reflection), } \\ -w^{*}<x^{*}<w^{*}, & \text { (capture), } \\ x^{*}>w^{*}, & \text { (transmission). }\end{cases}
$$

In Fig. 11(a) two regions may be clearly identified. Over one region, where roughly $\gamma_{0}<4$, only reflection is observed. Over the other, roughly $\gamma_{0}>40$, all events are of the transmission type. In between, the local phase variations seem to play an important role and subdivide the range $4<\gamma_{0}<40$ into little islands where all three phenomena alternate. This is made more evident in Fig. 11(b) where we zoom in on a small part of the mixed region. Note the sequence of events in Fig. 11(b): reflection $\rightarrow$ capture $\rightarrow$ transmission $\rightarrow$ capture $\rightarrow$ reflection. The whole sequence results when $\gamma_{0}$ is varied between 21.2 and 22.8 only. Thus, if one is to increase $\gamma_{0}$ from 21 to 22 in a single step, all the intervening capture and transmission events will be missed. In the data used to produce Fig. 11(b), $\gamma_{0}$ is fine-tuned in steps of size 0.001. Thus over the range of incident kinetic energies covered by Fig. 11(b), the ratio $K / U_{p}$ takes values between 0.808 and 0.803. In other words, although the kinetic energy is still less than the ponderomotive potential energy over the whole range, we have subranges of reflection as well as capture and transmission occurring even more than once. The message here is that approximate criteria, based on the ponderomotive model, may be arrived at but they cannot be relied on to sharply distinguish between the three phenomena of reflection, capture, and transmission. One still can safely state that reflection clearly occurs for $K \ll U_{p}$ and transmission is sure to result for $K \gg$ $U_{p}$, as has been demonstrated in Fig. 11(a). Finally, note that setting $K=U_{p}$ results in $\gamma_{0}=1+q^{2} / 4=26$, i.e., at about the middle of the mixed region for the case at hand.

\section{EXTRACTING THE ELECTRON FROM THE BEAM}

We have presented ample evidence for the capture and subsequent acceleration of low energy electrons by a laser beam. It has been demonstrated that a net gain results when the electron is injected into points on the beam axis on both sides of the focal point. In those regions the fields are rendered asymmetric by the process of focusing and impart momentum to the injected electron in the form of a strong impulse. By contrast, close to the focus the fields are approximately plane wave in character, and as such they present the electron with conditions obeying the Lawson-Woodward theorem [30]. Thus motion there may be oscillatory and a net gain may not be so great (consider the case corresponding to $\gamma_{0}=15$ in Fig. 6). 
A question that immediately comes to mind concerns getting the electron out of the beam while it still retains part or all of the energy gained. Our calculations show that a magnetic field, pointing in the negative $y$ direction (into the page), may be used to do just that. The field can be static or pulsed and it may lead to a decrease or increase in the energy gain. The idea is that the addition of the externally applied magnetic field breaks the symmetry of the laser fields even further and constitutes a further violation of the conditions of the LawsonWoodward theorem. The net result is that the symmetry of stimulated emission and absorption gets destroyed and an extra amount of energy gain (see Fig. 12) or loss [18] becomes possible.

For our, mainly illustrative, purposes in this work, we have used a field of strength 2.5 T over a region of width $\Delta z=8 \mathrm{~cm}$ in order to produce the trajectories and gains shown in Fig. 12, guided by the hindsight gained from

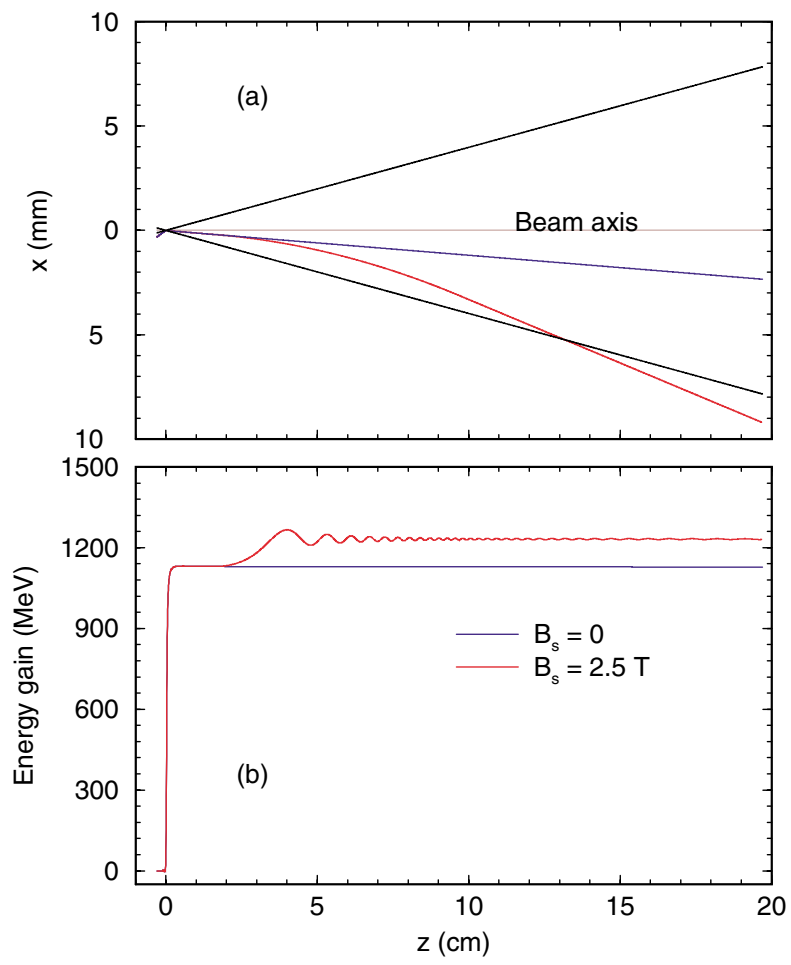

FIG. 12. (Color) Ejection of a captured electron from the laser beam. (a) The trajectories, and (b) the energy gain, of an electron injected with $\gamma_{0}=8.11$ and $\theta_{i}=6^{\circ}$ towards a point $z_{r} / 5$ to the right of the beam focus from a point with $z_{0}=$ $-3 \mathrm{~mm}$. The beam parameters are output power $P=10 \mathrm{PW}$ $(q \sim 86), w_{0}=8 \mu \mathrm{m}, \lambda=1 \mu \mathrm{m}$, and $\psi_{0}=0$. In this example, a uniform magnetic field of strength $B_{s}=2.5 \mathrm{~T}$ and pointing in the $-y$ direction (see Fig. 1) is used to eject the electron out of the beam. The total time of interaction is such that $\omega t=4 \pi \times$ $10^{5}$ and $B_{s}$ is on only in the region $1.7<z<9.7 \mathrm{~cm}$ covered during the interval $0.1 T_{i}<\Delta t<0.5 T_{i}$, where $T_{i}$ is the total interaction time. In (a) the dark black lines mark the beam boundaries as defined in Sec. IV. The legends in (b) apply to (a) as well.
Fig. 10. This static magnetic field is enough to bend the electron trajectory and get it out, in the sense described above, nearly $13 \mathrm{~cm}$ to the right of the beam focus. As is evident from Fig. 12(b) the final energy gain is increased from 1128 to $1230 \mathrm{MeV}$, or by about $9 \%$, during this process. This sudden change of direction, facilitated by the uniform magnetic field, puts the electron abruptly on a collision course with the many photons that would otherwise stream by and leave it cruising in the same direction, apart perhaps from a relatively small number of stimulated Compton events. A sudden increase in the electron's momentum is a result of the many inelastic Compton scattering events it undergoes as it changes course and escapes.

We note here that the point of extraction has been decided, in all of our calculations, simply by letting the electron interact with the fields for a time long enough for the net energy gain to cease to change and yet short enough for the calculation not to take so long to complete. Recall also that extraction is practically easier far away from the focal point, where the intensity drops markedly and concern about damage of the optical elements becomes less severe. It is also desirable to keep the distance over which the magnetic field is applied as small as possible.

Extraction using a static magnetic field need not be the only mechanism. In a realistic accelerator design, a beam of electrons would normally be injected for acceleration, and the accelerating laser beam can then be deflected away from the electron beam path by means of a judiciously placed parabolic mirror [31]. The accelerated electrons may be made to exit through a hole in the mirror. Needless to say that that calls for the use of a mirror with a damage threshold high enough to withstand the high laser intensities we are talking about.

\section{EFFECT OF A LASER BANDWIDTH}

Inherent in our model is the assumption of the fields being monochromatic. This is, admittedly, an idealization, for a tightly focused laser beam tends to develop a bandwidth. The resulting frequency variations cause sensitive local phase variations which, in turn, affect the energy gains quite sensitively. To demonstrate this point we have calculated the gain under conditions the same as those of Fig. 12 and for a band of frequencies of width $\Delta \nu=1.1996 \times 10^{12} \mathrm{~Hz}$ centered on $\nu=c / \lambda=$ $3 \times 10^{14} \mathrm{~Hz}$. The gain vs frequency is shown in Fig. 13 for the two laser power outputs we have been using all along. Note that high energy gains occur over bands of frequencies separated by other bands (gaps) that correspond to little or no gains at all. Furthermore, the gain bands (gaps) are narrower (wider) the higher the laser power output, and vice versa. 


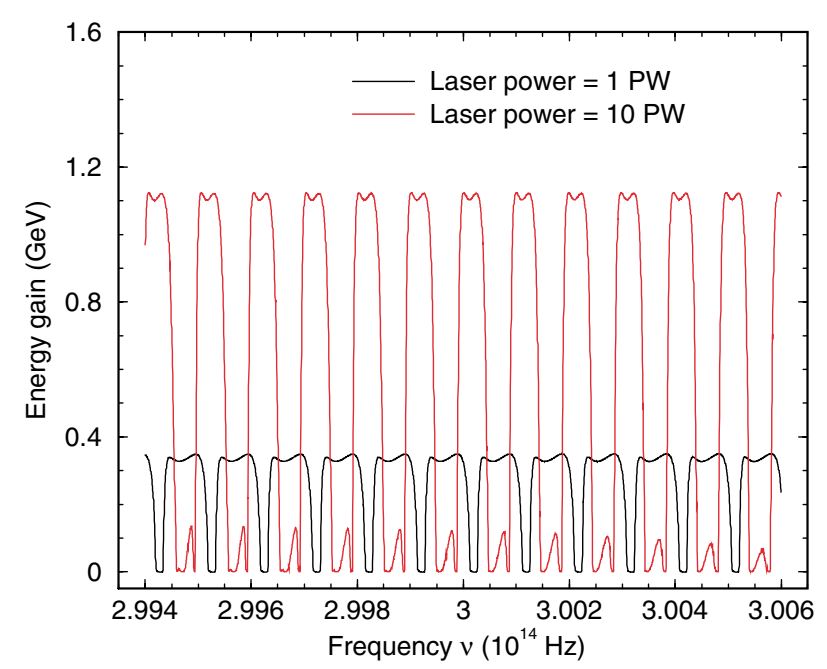

FIG. 13. (Color) Energy gain vs the laser frequency. The laser parameters and initial conditions on the electron injection are the same as in Fig. 12.

\section{SUMMARY AND CONCLUSIONS}

Most treatments of laser-electron acceleration that model the radiation field in terms of plane waves help to demonstrate acceleration only in principle. Lowest-order Gaussian beams model the intense fields, that may lead to sizable electron acceleration, much better, but also ignore corrections that may otherwise alter the electron dynamics quite appreciably. We have presented an investigation based on a representation of the fields of a Gaussian beam in which up to and including fifth-order corrections to the various electric and magnetic laser fields have been retained. The complexity of such fields, however, renders an analytic solution to the electron's equations of motion almost impossible.

We have solved the equations numerically, employing corrections to the fields of up to the order of 5 in the diffraction angle. Using our solutions, we have investigated a number of issues concerning the injection, acceleration, and extraction of a single electron in the fields of the linearly polarized Gaussian beam. Our conclusions may be summarized as follows. It is relatively easy for an energetic electron to pierce through the small beam focus. When an electron is injected at an angle to the beam axis, it mainly passes through with little deviation from the initial direction of motion and with very little gain or loss of energy. In some cases, especially when the injection energy is not great enough, the electron may be captured or reflected, again with some energy gain. However, if aimed at a point slightly to the right of the focus, the electron is captured and accelerated to high energy almost always. It is much less probable for such an electron to be reflected or transmitted. Captured electrons may be extracted from the accelerating beam by application of a laboratory uniform magnetic field. The added uniform magnetic field may cause the electron to lose or gain additional energy.

\section{ACKNOWLEDGMENTS}

Y. I. S. gratefully acknowledges support for this work from the German DAAD Gastdozentenprogramm. He also thanks the Palestinian National Institute for Information Technology (NIIT) in Ramallah for hospitality where part of this work was done. G. R. M. and C. H. K. are funded by the German Science Foundation (Nachwuchsgruppe within SFB 276).

\section{APPENDIX: FIELDS NEAR THE FOCUS OF A PULSED GAUSSIAN BEAM}

We briefly outline here the derivation of the electric and magnetic fields near the focus of a pulsed Gaussian beam following the procedure given by McDonald [27], which in turn proceeds along lines similar to those of Davis [24] and Barton and Alexander [25]. The beam axis will be taken along $z$ and its focus at $z=0$.

The fields will be assumed to have harmonic time dependence that goes like $e^{i \omega t}$. On the other hand, we follow McDonald in employing a pulse-shape function $g(\eta)$, where $\eta=\omega t-k z$, subject to a restriction to be encountered below. The fields will be derived from the linearly polarized vector potential

$$
\boldsymbol{A}=\hat{x} A_{0} g(\eta) \Psi(\mathbf{r}) e^{i \eta},
$$

where $A_{0}$ is a constant amplitude. The vector potential satisfies the following wave equation:

$$
\nabla^{2} \boldsymbol{A}-\frac{1}{c^{2}} \frac{\partial^{2} \boldsymbol{A}}{\partial t^{2}}=0
$$

provided the Lorentz gauge condition,

$$
\frac{1}{c} \frac{\partial \phi}{\partial t}+\nabla \cdot A=0
$$

is simultaneously satisfied. Direct substitution, followed by some algebra, leads to

$$
\nabla^{2} \Psi-2 i k \frac{\partial \Psi}{\partial z}\left(1-i \frac{g^{\prime}}{g}\right)=0
$$

where $g^{\prime}$ stands for a single differentiation with respect to $\eta$. As suggested by McDonald [23], we will look for a pulse-shape function that satisfies the condition

$$
g^{\prime} \ll g .
$$

This condition will be used repeatedly in the derivations to be carried out below. Next, we introduce a rescaling of the coordinates by letting

$$
\xi=\frac{x}{w_{0}}, \quad v=\frac{y}{w_{0}}, \quad \zeta=\frac{z}{z_{r}}, \quad z_{r}=\frac{k w_{0}^{2}}{2},
$$


where $w_{0}$ is the radius of the beam waist at focus and $z_{r}$ is the Rayleigh length. With these transformations Eq. (A4) becomes

$$
\nabla_{\perp}^{2} \Psi-4 i \frac{\partial \Psi}{\partial \zeta}+\epsilon^{2} \frac{\partial^{2} \Psi}{\partial \zeta^{2}}=0
$$

where

$$
\nabla_{\perp}^{2}=\frac{\partial^{2}}{\partial \xi^{2}}+\frac{\partial^{2}}{\partial v^{2}} ; \quad \epsilon=\frac{w_{0}}{z_{r}}=\frac{\lambda}{\pi w_{0}} .
$$

$\epsilon$ is the diffraction angle. Note that $\epsilon^{2}$ is small and may thus be used as an expansion parameter for a form of $\Psi(\xi, v, \zeta)$. Hence, we try

$$
\Psi=\Psi_{0}+\epsilon^{2} \Psi_{2}+\epsilon^{4} \Psi_{4}+\cdots .
$$

With this substitution, Eq. (A7) splits into

$$
\begin{gathered}
\nabla_{\perp}^{2} \Psi_{0}-4 i \frac{\partial \Psi_{0}}{\partial \zeta}=0, \\
\nabla_{\perp}^{2} \Psi_{2}-4 i \frac{\partial \Psi_{2}}{\partial \zeta}+\frac{\partial^{2} \Psi_{0}}{\partial \zeta^{2}}=0, \\
\nabla_{\perp}^{2} \Psi_{4}-4 i \frac{\partial \Psi_{4}}{\partial \zeta}+\frac{\partial^{2} \Psi_{2}}{\partial \zeta^{2}}=0,
\end{gathered}
$$

These equations have exact solutions. It can be shown, by direct substitution, that

$$
\begin{gathered}
\Psi_{0}=f e^{-f \rho^{2}}, \\
\Psi_{2}=\left(\frac{f}{2}-\frac{f^{3} \rho^{4}}{4}\right) \Psi_{0},
\end{gathered}
$$

$$
\Psi_{4}=\left(\frac{3 f^{2}}{8}-\frac{3 f^{4} \rho^{4}}{16}-\frac{f^{5} \rho^{6}}{8}+\frac{f^{6} \rho^{8}}{32}\right) \Psi_{0}
$$

where

$$
f=\frac{i}{\zeta+i}=\frac{e^{i \tan ^{-1} \zeta}}{\sqrt{1+\zeta^{2}}} ; \quad \rho^{2}=\xi^{2}+v^{2}
$$

$\Psi_{0}$ leads to the lowest-order Gaussian beam solution of the fields, $\Psi_{2}$ is the correction to order $\epsilon^{2}$, and so on. Assuming that the scalar potential has the same general structure as that of the vector potential, i.e.,

$$
\phi \sim g(\eta) \Phi(\mathbf{r}) e^{i \eta},
$$

then the Lorentz gauge (A3) yields

$$
\frac{\partial \phi}{\partial t}=i \omega \phi\left(1-i \frac{g^{\prime}}{g}\right) \approx i \omega \phi
$$

Hence,

$$
\phi=\frac{i}{k} \boldsymbol{\nabla} \cdot \boldsymbol{A} .
$$

Finally, the fields may be derived from

$$
\begin{gathered}
\boldsymbol{E}=-i k \boldsymbol{A}-\frac{i}{k} \boldsymbol{\nabla}(\boldsymbol{\nabla} \cdot \boldsymbol{A}), \\
\boldsymbol{B}=\boldsymbol{\nabla} \times \boldsymbol{A} .
\end{gathered}
$$

Dropping all terms of order $\epsilon^{6}$ and higher from Eq. (A9), we now write

$$
\begin{gathered}
\boldsymbol{A} \approx \hat{\boldsymbol{x}} A\left\{1+\boldsymbol{\epsilon}^{2}\left[\frac{f}{2}-\frac{f^{3} \rho^{4}}{4}\right]+\boldsymbol{\epsilon}^{4}\left[\frac{3 f^{2}}{8}-\frac{3 f^{4} \rho^{4}}{16}-\frac{f^{5} \rho^{6}}{8}+\frac{f^{6} \rho^{8}}{32}\right]\right\}, \\
A=A_{0} g(\eta) \Psi_{0} e^{i \eta} .
\end{gathered}
$$

This permits us to write the complex electric field components as

$$
\begin{gathered}
E_{x}=-i k A\left\{1+\epsilon^{2}\left[f^{2} \xi^{2}-\frac{f^{3} \rho^{4}}{4}\right]+\epsilon^{4}\left[\frac{f^{2}}{8}-\frac{f^{3} \rho^{2}}{4}-\frac{f^{4}}{16}\left(\rho^{4}-16 \xi^{2} \rho^{2}\right)-\frac{f^{5}}{8}\left(\rho^{6}+2 \xi^{2} \rho^{4}\right)+\frac{f^{6} \rho^{8}}{32}\right]+\cdots\right\}, \\
E_{y}=-i k A\left\{\epsilon^{2}\left[f^{2}\right] \xi v+\epsilon^{4}\left[f^{4} \rho^{2}-\frac{f^{5} \rho^{4}}{4}\right] \xi v+\cdots\right\}, \\
E_{z}=k A\left\{\epsilon[f] \xi+\epsilon^{3}\left[-\frac{f^{2}}{2}+f^{3} \rho^{2}-\frac{f^{4} \rho^{4}}{4}\right] \xi+\epsilon^{5}\left[-\frac{3 f^{3}}{8}-\frac{3 f^{4} \rho^{2}}{8}+\frac{17 f^{5} \rho^{4}}{16}-\frac{3 f^{6} \rho^{6}}{8}+\frac{f^{7} \rho^{8}}{32}\right] \xi+\cdots\right\} .
\end{gathered}
$$

On the other hand, the complex magnetic field components are

$$
B_{x}=0 \text {, }
$$




$$
\begin{gathered}
B_{y}=-i k A\left\{1+\epsilon^{2}\left[\frac{f^{2} \rho^{2}}{2}-\frac{f^{3} \rho^{4}}{4}\right]+\epsilon^{4}\left[-\frac{f^{2}}{8}+\frac{f^{3} \rho^{2}}{4}+\frac{5 f^{4} \rho^{4}}{16}-\frac{f^{5} \rho^{6}}{4}+\frac{f^{6} \rho^{8}}{32}\right]+\cdots\right\}, \\
B_{z}=k A\left\{\epsilon[f] v+\epsilon^{3}\left[\frac{f^{2}}{2}+\frac{f^{3} \rho^{2}}{2}-\frac{f^{4} \rho^{4}}{4}\right] v+\epsilon^{5}\left[\frac{3 f^{3}}{8}+\frac{3 f^{4} \rho^{2}}{8}+\frac{3 f^{5} \rho^{4}}{16}-\frac{f^{6} \rho^{6}}{4}+\frac{f^{7} \rho^{8}}{32}\right] v+\cdots\right\} .
\end{gathered}
$$

The real parts of these expressions give rise to the physical fields, Eqs. (6)-(11), that enter into the calculations.

*Permanent address: Physics Department, Birzeit University, P.O. Box 14, Birzeit, West Bank, Palestine. Electronic address: ysalamin@birzeit.edu

[1] M. D. Perry et al., Opt. Lett. 24, 160 (1999).

[2] J. Andruszkow et al., Phys. Rev. Lett. 85, 3825 (2000).

[3] Th. Stöhlker et al., Phys. Rev. Lett. 86, 983 (2001).

[4] R. Kienberger et al., Science 2971144 (2002).

[5] B. Hafizi, A. Ting, E. Esarey, P. Sprangle, and J. Krall, Phys. Rev. E 55, 5924 (1997).

[6] B. Hafizi, A. K. Ganguly, A. Ting, C. I. Moore, and P. Sprangle, Phys. Rev. E 60, 4779 (1999).

[7] J. X. Wang, Y. K. Ho, Q. Kong, L. J. Zhu, L. Feng, W. Scheid, and H. Hora, Phys. Rev. E 58, 6575 (1998).

[8] J. X. Wang, Y. K. Ho, L. Feng, Q. Kong, P. X. Wang, Z. S. Yuan, and W. Scheid, Phys. Rev. E 60, 7473 (1999).

[9] L. J. Zhu, Y. K. Ho, J. X. Wang, and L. Feng, J. Phys. B 32, 939 (1999).

[10] Q. Kong, Y. K. Ho, J. X. Wang, P. X. Wang, L. Feng, and Z. S. Yuan, Phys. Rev. E 61, 1981 (2000).

[11] Y. Chang and Z. Xu, Appl. Phys. Lett. 74, 2116 (1999).

[12] Y. I. Salamin and C. H. Keitel, Appl. Phys. Lett. 77, 1082 (2000).

[13] Y. I. Salamin and F. H. M. Faisal, Phys. Rev. A 61, 043801 (2000).

[14] Y. I. Salamin, F. H. M. Faisal, and C. H. Keitel, Phys. Rev. A 62, 53809 (2000).

[15] G. Malka and J. L. Miquel, Phys. Rev. Lett. 77, 75 (1996).

[16] G. Malka, E. Lefebvre, and J. L. Miquel, Phys. Rev. Lett. 78, 3314 (1997).
[17] P. X. Wang, Y. K. Ho, X. Q. Yuan, Q. Kong, N. Cao, A. M. Sessler, E. Esarey, and Y. Nishida, Appl. Phys. Lett. 78, 2253 (2001).

[18] Y. I. Salamin and C. H. Keitel, Phys. Rev. Lett. 88, 095005 (2002).

[19] E. S. Sarachik and G. T. Schappert, Phys. Rev. D 1, 2738 (1970).

[20] F.V. Hartemann, S. N. Fochs, G. P. Le Sage, N.C. Luhmann, Jr., J. G. Woodworth, M. D. Perry, Y. J. Chen, and A. K. Kerman, Phys. Rev. E 51, 4833 (1995).

[21] Y. I. Salamin and F. H. M. Faisal, Phys. Rev. A 54, 4383 (1996).

[22] Y. I. Salamin and F. H. M. Faisal, J. Phys. A 31, 1319 (1998).

[23] K. McDonald and K. Shmakov, Phys. Rev. ST Accel. Beams 2, 121301 (1999).

[24] L.W. Davis, Phys. Rev. A 19, 1177 (1979).

[25] J. P. Barton and D. R. Alexander, J. Appl. Phys. 66, 2800 (1989).

[26] M. O. Scully and M. S. Zubairy, Phys. Rev. A 44, 2656 (1991).

[27] K. T. McDonald, www.hep.princeton.edu/ mcdonald/ accel/gaussian.ps; www.hep.princeton.edu/ mcdonald/ accel/gaussian2.ps.

[28] F.V. Hartemann, J. R. Van Meter, A. L. Troha, E. C. Landahl, N. C. Luhmann, Jr., H. A. Baldis, Atul Gupta, and A. K. Kerman, Phys. Rev. E 58, 5001 (1998).

[29] T.W. Kibble, Phys. Rev. 138, B740 (1965).

[30] For a review, see E. Esarey, P. Sprangle, and J. Krall, Phys. Rev. E 52, 5443 (1995).

[31] J. L. Hirshfield and C. Wang, Phys. Rev. E 61, 7252 (2000). 